\title{
New Low Temperature Sterilization Processes
}

\author{
Gerald McDonnell ${ }^{1}$, Peter A. Burke ${ }^{2}$ \\ ${ }^{1}$ STERIS Ltd, Basingstoke, United Kingdom \\ ${ }^{2}$ STERIS Corporation, Ohio, USA.
}

doi: 10.3396/ijic.V5i2.021.09

It is an exciting time in surgical procedure development and instrument design. In parallel with the many advances in minimum invasive surgical techniques, the instruments used are becoming more complex, with integrated electronics, robotic control and precise operating mechanisms. Many of these contain a variety of temperature-sensitive materials and can not be exposed to traditional, heat-based disinfection and sterilization techniques. Low temperature sterilization is increasingly desired to reprocess these types of devices in a microbial effective, device compatible and safe way. The requirements for all sterilization processes are changing, to include new standards (such as ISO 14337 Sterilization of health care products - General requirements for characterization of a sterilizing agent and the development, validation and routine control of a sterilization process), the requirement for validation, record-keeping, faster-turn around time, environmental concerns and reducing costs. In addition to traditional test organisms such as bacteria and bacterial spores, these processes may also be expected to reduce the risks posed by emerging pathogens as well as other patient risks (such as the presence of toxins or 'infectious' proteins such as prions). Steam and dry heat sterilization have traditionally been the most widely used methods, but are restricted to temperature-sensitive materials and instruments. ${ }^{1}$ In the case of steam, the overall costs of installation, correct operation, maintenance and validation can be expensive (e.g., considering water, steam quality and drainage requirement). The traditional low temperature sterilization techniques based on humidified ethylene oxide and steamformaldehyde are not frequently used in hospitals, particularly due to longer overall reprocessing times, and safety concerns. More recent developments in low temperature sterilization included gas plasma systems, humidified ozone and liquid chemical peracetic acid. ${ }^{1}$

There are a number of new systems recently developed that all use hydrogen peroxide gas for low temperature sterilization. These can be considered as those using plasma as part of the process and those that only use hydrogen peroxide gas (Figure 1).

All these systems expose loads to be sterilized under vacuum to the gas, in the presence or absence of plasma. A plasma is essentially an excited gas and is produced by adding energy (in the form of heat or an electromagnetic field). The first plasma sterilizers used a series of pulses including saturated hydrogen peroxide gas and plasma generation for sterilization. In a new process, such as those promoted under the name of STERRAD NX and $100 \mathrm{NX}$, hydrogen peroxide gas is concentrated (by removal of water) and applied at very high concentrations to the load; following peroxide exposure, a plasma is generated 

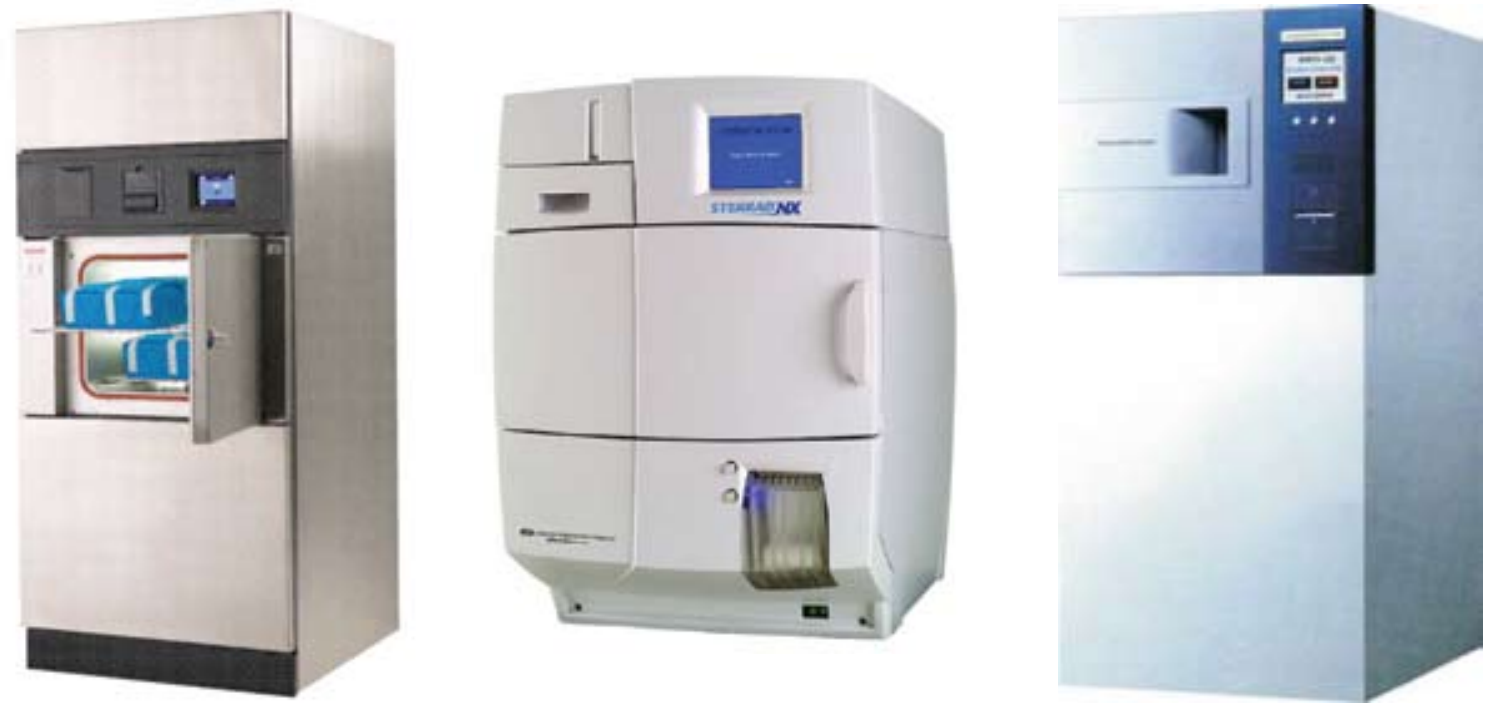

Figure 1: Examples of new low temperature sterilization processes based on hydrogen peroxide gas. The V-Pro1 (left) uses peroxide gas alone, while the STERRAD NX (middle) and HMTS 142 (right) both use plasma as part of the process.

that aids in the removal of peroxide residuals from the load. ' In some plasma systems, such as the HMTS 142, the plasma is claimed to be used during each exposure phase with hydrogen peroxide. In contrast, the V-Pro 1 process is a simple vacuum-based process uses only hydrogen peroxide gas for sterilization and does not include any plasma. It is important to note that each peroxide gas-based system can vary in exposure conditions (e.g., concentration of gas, saturated or unsaturated gas, presence/absence of plasma etc), and therefore should be considered separately for efficacy, compatibility and safety. Further consideration in this report is given to the V-Pro 1, that uses hydrogen peroxide under vacuum in a defined process.

Hydrogen peroxide is one of the most widely used biocides for various antiseptic, disinfectant and sterilization application. 'When in the gas phase, hydrogen peroxide (also known as VHP for Vaporized Hydrogen Peroxide) demonstrates significantly greater antimicrobial efficacy and compatibility than in the liquid form. This may be due to the more reactive nature of the gas but recent evidence would also suggest that the mechanism of action of the gas is different to the liquid. Gaseous peroxide is not a new technology, being used for over 15 years for low temperature disinfection and sterilization applications, in particular in high risk pharmaceutical and medical device manufacturing. ${ }^{2}$ The technology is new to clinical applications, already being used for environmental disinfection (under atmospheric pressure) and now as a rapid, low temperature vacuum sterilization process. Gaseous hydrogen peroxide has been confirmed to have broad spectrum antimicrobial activity with confirmedvirucidal, bactericidal, fungicidal, mycobactericidal, fungicidal, cysticidal and sporicidal activity. Antimicrobial activity has been confirmed in the presence of interfering soils, with activity dependant on the gas concentration and exposure time. ${ }^{3}$ In addition, recent reports have confirmed that peroxide gas is also effective against various strains of prions, the agents responsible for Transmissible Spongiform Encephalopathies such as Creutzfeldt-Jakob Disease. ${ }^{4}$ Mode of action studies indicate that hydrogen peroxide gas has different mechanisms of action in comparison to liquid peroxide; the gas, as an oxidizing agent, has been particularly shown to attack and breakdown the various macromolecules that make up cellular and viral structure such as proteins, lipids and nucleic acids. ${ }^{1,2}$ From these studies, the mode of action of liquid peroxide (including when present under saturated gas conditions) appears to be distinctly different.

Hydrogen peroxide gas also offers further advantages. In addition to its rapid antimicrobial activity, it provides a balance of material compatibility and safety in comparison to other gaseous oxidizing agents. In gas form, hydrogen peroxide has been shown to have compatibility with the most commonly used metals 


\section{V-PRO 1 Sterilization Cycle}

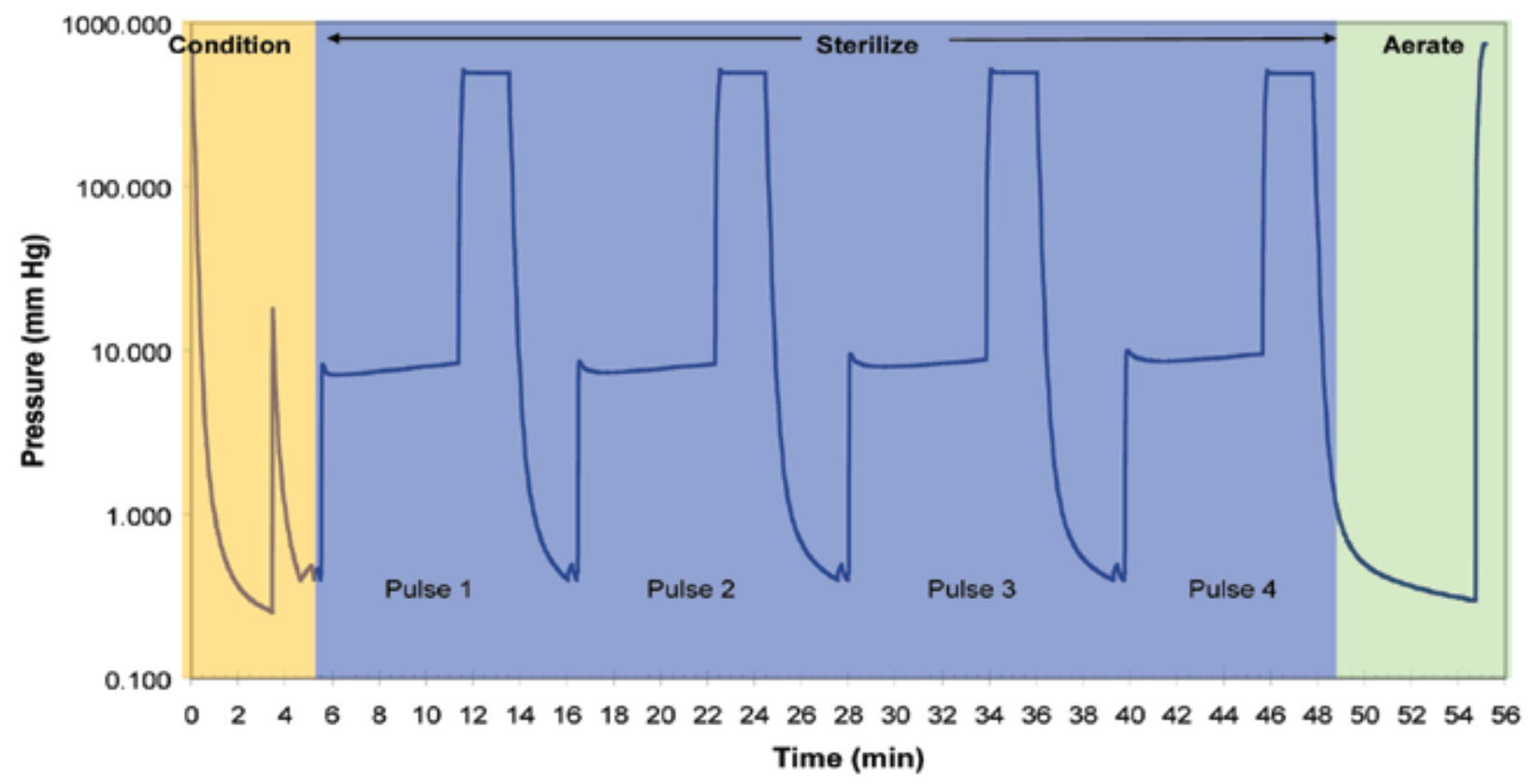

Figure 2. A typical gaseous hydrogen peroxide sterilization Cycle. This graph shows the pressure changes during the process, consisting of three phases (conditioning, sterilization and aeration). Note that atmospheric pressure is $760 \mathrm{mmHg}$ or $101.35 \mathrm{kPa}$.

and plastics in instruments and, when controlled in the 'dry' gas phase can even be compatible with electrical components. The gas can be safely contained, easily degraded/removed during the process and rapidly degrades into water and oxygen, providing an important environmental benefit when utilized under vacuum conditions. Practically, these systems require little installation requirements (electricity only) in comparison to other low and high temperature alternatives, rapid cycle time $(<1$ hour $)$ and provides sterile instruments for immediate use or sterile storage (with no extended aeration requirements as traditionally needed with ethylene oxide and formaldehyde). There are, however, some limitations in that instruments should be provided dry for sterilization, the process can not be used for liquids and cellulose-based materials (such as paper), and some studies have shown limited life for Nylon-containing materials over multiple cycles.

This newer systems is particularly intended for the terminal sterilization of cleaned, rinsed, and dried, reusable metal and non-metal medical devices used in healthcare facilities. The sterilization cycle consists of three phases (Figure 2): conditioning, sterilization and aeration. The overall process is conducted under vacuum to ensure air removal and sterilant gas penetration, but is also a safety feature containing the gas during the process. For operation, liquid hydrogen peroxide (at $59 \%$ in water) is provided in a sealed container and used to generate the gas within the sterilization chamber. At completion of the cycle, the load is removed and can be immediately used or stored prior to use.

International standards have been published that describe the minimal requirements for any sterilization process, such as those based on steam, ethylene oxide and any new process. ISO EN 14937 specifically defines the characterization of any sterilizing agent and the development, validation and routine control of its safe and effective use within a defined process. There are a minimum of three considerations: antimicrobial efficacy, compatibility and safety. In addition to demonstrated broad spectrum activity, the process has been verified to provide a minimum sterility assurance level (SAL) of $10^{-6}$ (by overkill demonstration) with Geobacillus stearothermophilus endospores being the most-resistant organism. Process validation included the demonstration of device 
sterilization under simulative (laboratory) and hospital (in use) conditions. Safety considerations and testing have included requirements for patient safety (being non-toxic following sterilization under ISO 10993-17), device compatibility (material, component and full device testing), staff safety (ensuring the absence of the gas in the area of the sterilizer during normal use) and the environment. In line with international standards, the sterilizer contains an integrated, independent safety monitoring system that ensures the process is conducted within specification each and every cycle.

Now and in the future we expect to see further advances in surgical and general instrument design, allowing for greater surgical flexibility and patient outcomes. As these advances continue, the reprocessing (cleaning, disinfection and sterilization) practices need to be able to safely handle these instruments in a safe and effective manner. These should optimally consider both microbial and non-microbial agents, to ensure infection prevention and patient/staff safety. Cost and inventory-control considerations in hospitals will require close consideration, as well as the requirements for tracking, traceability and evidencebased verification of reprocessing safety. With parallel advances in instrument technology and reprocessing methods these challenges can be met in a safe, effective and practical manner.

\section{References}

1. McDonnell G. Antisepsis, Disinfection, and Sterilization: Types, Action, and Resistance. Washington DC ASM Press, 2007.

2. McDonnell G. Peroxygens and other forms of oxygen. Their use for effective cleaning, disinfection and sterilization. In: Zhu PC Ed. Biocides old and new: where chemistry and microbiology meet. ACS Symposium Series, Oxford University Press, New York, 2006.

3. Meszaros JE, Antloga K, Justi C, et al. Area Fumigation with Hydrogen Peroxide Vapor. Applied Biosafety 2005; 10(2): 91 100.

4. Fichet G, Antloga K, Comoy E, et al. Prion inactivation using a new gaseous hydrogen peroxide sterilisation process. Journal of Hospital Infection 2007; 67: 278-386. 